\title{
Endometrial Cancer and Hypermethylation: Regulation of DNA and MicroRNA by Epigenetics
}

\author{
Kouji Banno, Iori Kisu, Megumi Yanokura, Kenta Masuda, Yusuke Kobayashi, Arisa Ueki, \\ Kosuke Tsuji, Wataru Yamagami, Hiroyuki Nomura, Nobuyuki Susumu, and Daisuke Aoki \\ Department of Obstetrics and Gynecology, Keio University School of Medicine, Shinanomachi 35 Shinjuku-ku, Tokyo 160-8582, Japan \\ Correspondence should be addressed to Kouji Banno, kbanno@sc.itc.keio.ac.jp
}

Received 31 October 2011; Accepted 2 February 2012

Academic Editor: Yong-yu Liu

Copyright () 2012 Kouji Banno et al. This is an open access article distributed under the Creative Commons Attribution License, which permits unrestricted use, distribution, and reproduction in any medium, provided the original work is properly cited.

\begin{abstract}
Endometrial cancer is the seventh most common cancer in women worldwide. Therefore elucidation of the pathogenesis and development of effective treatment for endometrial cancer are important. However, several aspects of the mechanism of carcinogenesis in the endometrium remain unclear. Associations with genetic variation and mutations of cancer-related genes have been shown, but these do not provide a complete explanation. Therefore, in recent years, epigenetic mechanisms that do not involve changes in DNA sequences have been examined. Studies aimed at detection of aberrant DNA hypermethylation in cancer cells present in microscopic amounts in vivo and application of the results to cancer diagnosis have also started. Breakdown of the DNA mismatch repair mechanism is thought to play a large role in the development of endometrial cancer, with changes in the expression of the $h M L H 1$ gene being particularly important. Silencing of genes such as APC and CHFR, Sprouty 2, RASSF1A, GPR54, CDH1, and RSK4 by DNA hypermethylation, onset of Lynch syndrome due to hereditary epimutation of $h M L H 1$ and hMSH2 mismatch repair genes, and regulation of gene expression by microRNAs may also underlie the carcinogenic mechanisms of endometrial cancer. Further understanding of these issues may permit development of new therapies.
\end{abstract}

\section{Introduction}

Endometrial cancer is the seventh most common cancer in women worldwide. In Japan, westernization of lifestyle has increased the number of patients with endometrial cancer, and this disease now accounts for about $40 \%$ of cancers of the uterus. A further increase, and a younger onset age are also predicted, and therefore elucidation of the pathogenesis and development of effective treatment are needed. However, the mechanism of carcinogenesis in the endometrium remains unclear. Genetic aberrances such as variations in gene expression and mutation of cancer-related genes have been identified, but these do not fully explain canceration in the endometrium. Therefore, epigenetic changes in gene expression through effects on chromatin without DNA mutation are drawing attention. Breakdown of the DNA mismatch repair mechanism by aberrant DNA hypermethylation is particularly important for development of type 1 endometrial cancer, and changes in expression of genes such as human MutL homolog1 (hMLH1) and human MutS homolog2 ( $h M S H 2)$ may be involved in this mechanism. The possible epigenetic mechanisms include epimutation, hypermethylation causing epimutation, and regulation of gene expression by small noncoding RNAs, called microRNAs, that bind upstream of the gene and do not change the methylation level of the gene itself. In this review, DNA hypermethylation associated with endometrial cancer based on epimutation of three genes, $h M L H 1, h M S H 2$ and epithelial cell adhesion molecule (EPCAM), and the effects of miRNAs in endometrial cancer will be discussed.

\section{New Findings on DNA Hypermethylation in Endometrial Cancer}

Among the epigenetic mechanisms, DNA methylation has been most widely studied. Changes in DNA methylation have been associated with various tumorous lesions $[1,2]$, and hypermethylation is commonly associated with downregulation of gene expression. Our review in 2009 [3] prompted 
further studies of aberrant DNA hypermethylation associated with endometrial cancer, and it has been found that hypermethylation of gene promoters is linked to reduced expression of several genes in this cancer. We have reported silencing due to hypermethylation for $h M L H 1$ and adenomatous polyposis coli (APC), E-cadherin, checkpoint with FHA and RING (CHFR), caspase-8 (CASP8), transforming growth factor, beta receptor II (TGF-bRII), $p 73$, homeobox A11 (HOXA11), and catechol-O-methyltransferase (COMT) [3], and genes such as Sprouty 2 [4], Ras association domain family 1 isoform A (RASSF1A) [5], G-protein coupled receptor 54 (GPR54) [6], cadherin 1 (CDH1) [7], and ribosomal S6 kinase4 (RSK4) [8] have also been found to be silenced by a similar mechanism. Hypermethylation of the APC promoter is not found in the normal endometrium or in endometrial hyperplasia but is detected in atypical hyperplasia and early endometrial cancer. Interestingly, the frequency of hypermethylation in the APC promoter is reduced with progression of endometrial cancer, which led Ignatov et al. to suggest that this hypermethylation may be an important event in early canceration of the endometrium [9]. Satoh et al. linked hypermethylation to the response of tumors to taxane drugs [10], and Wang et al. found that reduced expression of CHFR by hypermethylation improves the response of both stomach and endometrial cancers to paclitaxel [11]. These studies suggest the possibility of personalized cancer treatment adapted to each patient following examination of the expression levels of multiple genes.

Sprouty 2 (SPRY2) is an antagonist regulator of receptor tyrosine kinases in the fibroblast growth factor (FGF) and RAS-MAPK pathways and a tumor suppressor gene involved in cell proliferation, differentiation, and angiogenesis. Parts of the FGF and RAS-MAPK pathways are changed in endometrial cancer, and expression of SPRY2 is reduced due to hypermethylation in several types of cancer [12-14]. Velasco et al. found that SPRY2 in the normal endometrium is expressed in accord with the menstrual cycle and suggested that SPRY2 contributes to growth of glandular structures [4]. SPRY2 expression is extremely low in advanced invasive cancers and other types of endometrial cancer, other than endometrioid adenocarcinoma, which indicates that SPRY2 may play a role in suppression of endometrial cancer by regulating the MAPK pathway [4].

RASSF1A is a tumor suppressor that is a negative regulator in the RAS-MAPK pathway and, along with SPRY2, has reduced expression in various cancers. In a comparison of normal and cancerous endometrial tissues, Pallares et al. showed that RASSF1A promoter hypermethylation and reduced expression were particularly prevalent in endometrial cancer with microsatellite instability, especially in advanced cancers [5]. This led to the suggestion that RASSF1A participates in cell proliferation and apoptosis by regulating the MAPK pathway and has effects on canceration of the endometrium [5].

GPR54 is a gene-encoding endogenous receptor of kisspeptin (KISS1), a suppressor of cancer metastasis, and has reduced expression in some cancers, but an unclear status in endometrial cancer. Kang et al. found high survival rates in cases with high GPR54 expression and showed that expression of GPR54 is epigenetically regulated [6]. Functional recovery of GPR54 was possible with treatment with 5-aza-dC, a drug that causes DNA hypomethylation, and subsequent expression of GPR54 and activation of a downstream response pathway involving metastin-10 were effective for inhibiting metastasis of endometrial cancer [6].

$C D H 1$ is a promoter of E-cadherin. Yi et al. found that hypermethylation of $C D H 1$ reduced E-cadherin expression in endometrial cancer, with resulting effects on clinical and pathological progression and 5-year survival rates. Hypermethylation of $C D H 1$ is common in undifferentiated cancers, leading to the suggestion that this mechanism may be closely associated with canceration and invasive capacity [7].

X-linked Ribosomal S6 Kinase RPS6KA6 (RSK4) is a substrate of ERK that inhibits transcriptional activity of some receptor tyrosine kinases for certain targets. RSK4 is also associated with the FGFR2/RAS/ERK signal pathway and is a tumor suppressor. Dewdney et al. showed that expression of RSK 4 is reduced by hypermethylation in colon, breast, and kidney cancer, as well as in endometrial cancer, but the tumor inhibitory action of RSK4 in the endometrium is unclear [8].

\section{Epimutation and Carcinogenesis of the Endometrium}

Epimutation refers to the epigenetic silencing of a gene for which expression is normally not suppressed, or epigenetic activation of a gene for which expression is normally suppressed $[15,16]$. Studies of canceration of the endometrium and epimutation of genes have mainly focused on $h M L H 1, h M S H 2$, and EPCAM. hMLH1 and $h M S H 2$ are DNA mismatch repair (MMR) genes that have a strong association with endometrial cancer, above that of other MMR genes such as hMSH6 and PMS2 [17]. Kondo et al. first showed that epigenetic inhibition of $h M L H 1$ expression is more frequent than that of $h M S H 2$ in endometrial cancer [18]. Most subsequent studies have examined $h M L H 1$, and this gene has been found to be a tumor suppressor that has reduced expression in various cancers. Mutation of $h M L H 1$ is especially common in cases with multiple primary cancers such as, for example, in Lynch syndrome (hereditary nonpolyposis colorectal cancer: HNPCC), and epimutation of the germline may be present even if mutation of $h M L H 1$ itself does not occur [19]. Hitchins et al. suggested that the epimutation may be inherited through the mother since during egg formation an epigenetic error can essentially be solved by demethylation; however, this type of heredity epimutation occurs with lower frequency than the germline mutation [20]. However, in one of two cases in which epimutation of $h M L H 1$ occurred de novo, Goel et al. showed that the epimutation occurred in the allele derived from the father, rather than the mother [21]. This defect may have occurred during egg formation without demethylation, and the epimutation may have been inherited or may have occurred as a new epimutation after fertilization. 
An inherited germline epimutation of $h M S H 2$ was reported in 2006 by Chan et al. [22] in a family line of three patients (brothers and sisters) with colon or endometrial cancer with onset at an early age. Genetic mutation of hMSH2 was not present, but protein deficiency was recognized, and microsatellite instability was shown, with the epimutation of $h M S H 2$ inherited from the mother. The same epimutation was inherited by three children of the three patients, indicating that not only a DNA sequence mutation but also an epimutation can be inherited over multiple generations. Sequencing shows that the epimutation does not occur in all cells and methylation mosaics exist. Different degrees of methylation occur based on the two-hit theory and can be the cause of heritability of diseases.

The EPCAM gene codes for an epithelial cell adhesion molecule and is overexpressed in most cancers. There is a diversity of opinion on the effects of EPCAM on canceration. EPCAM is a homophilic intercellular adhesion molecule that may prevent metastasis, but conversely EPCAM may also promote metastasis of cancers to prevent intercellular adhesion mediated by E-cadherin [23]. In endometrial cancer, EPCAM deficiency is also involved in hypermethylation of the $h M S H 2$ promoter [24]. Ligtenberg et al. found that epimutation of EPCAM itself is not involved in development of endometrial cancer, but a mutation to the $3^{\prime}$ side of EPCAM upstream and close to $h M S H 2$ epigenetically silences hMSH2 and may lead to endometrial cancer and Lynch syndrome.

\section{Endometrial Cancer and Regulation with Aberrant Methylation of MicroRNA}

MicroRNAs (miRNAs) are short noncoding RNAs of about 18-25 bases that regulate expression of genes. miRNAs have been found to be downregulated by methylation of DNA in various cancers, and these miRNAs are referred to as tumor suppressor miRNAs (TS-miRNAs) [25, 26]. Identified TSmiRNAs include $m i R-124, m i R-126, m i R-137$, and $m i R-491$ [25-29]. Using a microarray assay, Huang et al. found that SRY-related high-mobility group box 4 (SOX4) genes were highly expressed in endometrial cancer cells. In database analysis, SOX4 was identified as a target for miR-129-2, and a reporter assay showed that $m i R-129-2$ was a negative regulator of SOX4. Expression of miR-129-2 was verified in 117 cases of endometrial cancer with increased expression of SOX4, and in $68 \%$ of the patients SOX4 expression was silenced by methylation. Histone acetylation and DNA demethylation were found to increase the expression of $m i R$ 129-2, reduce SOX4 expression, and suppress proliferation of cancer cells. Furthermore, hypermethylation of the miR-1292 gene was statistically related to microsatellite instability and the methylation status of $h M L H 1$ [30].

Expression of $m i R-152$ is also inhibited by DNA hypermethylation [31]. Tsuruta et al. identified $m i R-152$ as a candidate TS-miRNA in endometrial cancer through DNA methylation screening and expression screening of endometrial cancer cells. Methylation of $m i R-152$ is altered in acute lymphocytic leukemia, and miR-152 expression is changed in digestive system cancers and cholangiocellular cancer [32-34]. Tsuruta et al. found methylation and downregulation of miR-152 at high frequency and showed that expression of $m i R-152$ is recovered by 5 -azadC. Methylation of miR-152 is completely consistent with expression, and hypermethylation in the promoter region of miR-152 reduces the expression. DNMT1 is a well-known target of miR-152, and E2F3, MET, and Rictor have been identified as additional targets [34]. E2F3 is an E2F family transcriptional inhibitor and may be a cancer gene [35]. $M E T$ is a gene that encodes a cell-surface receptor for hepatocyte growth factor and a known cancer gene [36]. Rictor is a component of mTORC2 (mTOR complex 2$)$ which directly regulates phosphorylation of $A k t$ and is important in cancer cells $[37,38]$. Activation of $m$ TORC2-Akt signaling contributes to canceration of the endometrium, but the action of Rictor in canceration is unclear. However, silencing of miR-152 appears to lead to activation of multiple targets, and miR-152 itself and E2F3, MET, and Rictor may be new targets for treatment of endometrial cancer [31].

\section{Conclusion}

In 2007, endometrial cancer was newly diagnosed in as many as 226,000 women worldwide and is the cause of death of more than 7000 people annually in the USA alone. In Japan, the number of patients with this cancer is increasing and a younger age of onset is also predicted, which emphasizes the need to find more effective therapies based on improved understanding of the pathogenesis. As for many other cancers, development of endometrial cancer cannot be completely explained by genetic mutations alone and is likely to involve epigenetic changes. In endometrial cancer, mutations of MMR genes are important, and regulation of these genes has been widely examined. Expression of $h M L H 1, h M S H 2$, and EPCAM is suppressed by promoter hypermethylation, which inhibits direct or indirect DNA mismatch repair and contributes to development of endometrial cancer type 1 and development of endometrial cancer in Lynch syndrome. Reduced expression of genes such as APC, CHFR, Sprouty 2, RASSF1A, GPR54, CDH1, and RSK4 through a similar mechanism of hypermethylation has also been found in endometrial cancer. Suppression of gene expression by miRNAs also occurs in endometrial cancer, and expression of the miRNA itself may be increased or decreased by promoter methylation, based on differences between normal and cancerous endometrial tissue, and may contribute to canceration of the endometrium.

The studies described above have contributed to gradual elucidation of the mechanisms of canceration in the endometrium, but the findings do not seem to be applicable to all cases. Thus, it is unclear whether there is a common underlying mechanism or whether the apparently similar pathological conditions among cases of endometrial cancer are actually somewhat different and new classifications are needed. More knowledge of the canceration mechanism should reveal new therapeutic targets and discovery of new 
drugs. This is especially true for miRNA targeting since siRNA modulation of miRNA has been established in vitro and a similar approach in vivo may prevent progression of canceration caused by silencing of miRNA. Drugs that inhibit DNA methyltransferases and thus cause gene demethylation have also been developed, but improved targeting of these drugs is needed for practical clinical use.

\section{References}

[1] M. Esteller, "Epigenetics in cancer," New England Journal of Medicine, vol. 358, no. 11, pp. 1148-1159, 2008.

[2] S. A. Wajed, P. W. Laird, and T. R. DeMeester, "DNA methylation: an alternative pathway to cancer," Annals of Surgery, vol. 234, no. 1, pp. 10-20, 2001.

[3] Y. Muraki, K. Banno, M. Yanokura et al., "Epigenetic DNA hypermethylation: clinical applications in endometrial cancer," Oncology Reports, vol. 22, no. 5, pp. 967-972, 2009.

[4] A. Velasco, J. Pallares, M. Santacana et al., "Promoter hypermethylation and expression of sprouty 2 in endometrial carcinoma," Human Pathology, vol. 42, no. 2, pp. 185-193, 2011.

[5] J. Pallares, A. Velasco, N. Eritja et al., "Promoter hypermethylation and reduced expression of RASSF1A are frequent molecular alterations of endometrial carcinoma," Modern Pathology, vol. 21, no. 6, pp. 691-699, 2008.

[6] H. S. Kang, T. Baba, M. Mandai et al., "GPR54 is a target for suppression of metastasis in endometrial cancer," Molecular Cancer Therapeutics, vol. 10, no. 4, pp. 580-590, 2011.

[7] T. Z. Yi, J. Guo, L. Zhou et al., "Prognostic value of E-cadherin expression and $\mathrm{CDH} 1$ promoter methylation in patients with endometrial carcinoma," Cancer Investigation, vol. 29, no. 1, pp. 86-92, 2011.

[8] S. B. Dewdney, B. J. Rimel, P. H. Thaker et al., "Aberrant methylation of the X-linked ribosomal S6 kinase RPS6KA6 (RSK4) in endometrial cancers," Clinical Cancer Research, vol. 17, no. 8, pp. 2120-2129, 2011.

[9] A. Ignatov, J. Bischoff, T. Ignatov et al., "APC promoter hypermethylation is an early event in endometrial tumorigenesis," Cancer Science, vol. 101, no. 2, pp. 321-327, 2010.

[10] A. Satoh, M. Toyota, F. Itoh et al., "Epigenetic inactivation of CHFR and sensitivity to microtubule inhibitors in gastric cancer," Cancer Research, vol. 63, no. 24, pp. 8606-8613, 2003.

[11] X. Wang, Y. Yang, C. Xu et al., "CHFR suppression by hypermethylation sensitizes endometrial cancer cells to paclitaxel," International Journal of Gynecological Cancer, vol. 21, no. 6, pp. 996-1003, 2011.

[12] T. L. Lo, P. Yusoff, C. W. Fong et al., "The Ras/mitogenactivated protein kinase pathway inhibitor and likely tumor suppressor proteins, sprouty 1 and sprouty 2 are deregulated in breast cancer," Cancer Research, vol. 64, no. 17, pp. 61276136, 2004.

[13] H. Sutterlüty, C. E. Mayer, U. Setinek et al., "Down-regulation of Sprouty2 in non-small cell lung cancer contributes to tumor malignancy via extracellular signal-regulated kinase pathwaydependent and -independent mechanisms," Molecular Cancer Research, vol. 5, no. 5, pp. 509-520, 2007.

[14] C. W. Fong, M. S. Chua, A. B. McKie et al., "Sprouty 2, an inhibitor of mitogen-activated protein kinase signaling, is down-regulated in hepatocellular carcinoma," Cancer Research, vol. 66, no. 4, pp. 2048-2058, 2006.

[15] R. Holliday, "The inheritance of epigenetic defects," Science, vol. 238 , no. 4824 , pp. 163-170, 1987.
[16] P. N. Schofield, J. A. Joyce, W. K. Lam et al., "Genomic imprinting and cancer; new paradigms in the genetics of neoplasia," Toxicology Letters, vol. 120, no. 1-3, pp. 151-160, 2001.

[17] P. Peltomäki, "Lynch syndrome genes," Familial Cancer, vol. 4, no. 3, pp. 227-232, 2005.

[18] E. Kondo, T. Furukawa, K. Yoshinaga et al., "Not hMSH2 but hMLH1 is frequently silenced by hypermethylation in endometrial cancer but rarely silenced in pancreatic cancer with microsatellite instability." International journal of oncology, vol. 17, no. 3, pp. 535-541, 2000.

[19] C. M. Suter, D. I. K. Martin, and R. L. Ward, "Germline epimutation of MLH1 in individuals with multiple cancers," Nature Genetics, vol. 36, no. 5, pp. 497-501, 2004.

[20] M. P. Hitchins, J. J. L. Wong, G. Suthers et al., "Inheritance of a cancer-associated MLH1 germ-line epimutation," New England Journal of Medicine, vol. 356, no. 7, pp. 697-705, 2007.

[21] A. Goel, T. P. Nguyen, H. C. E. Leung et al., "De novo constitutional MLH1 epimutations confer early-onset colorectal cancer in two new sporadic Lynch syndrome cases, with derivation of the epimutation on the paternal allele in one," International Journal of Cancer, vol. 128, no. 4, pp. 869-878, 2011.

[22] T. L. Chan, S. T. Yuen, C. K. Kong et al., "Heritable germline epimutation of MSH2 in a family with hereditary nonpolyposis colorectal cancer," Nature Genetics, vol. 38, no. 10, pp. 1178-1183, 2006.

[23] B. T. F. van der Gun, L. J. Melchers, M. H. Ruiters, L. F. M. H. de Leij, P. M. J. McLaughlin, and M. G. Rots, "EpCAM in carcinogenesis: the good, the bad or the ugly," Carcinogenesis, vol. 31, no. 11, pp. 1913-1921, 2010.

[24] M. J. L. Ligtenberg, R. P. Kuiper, T. L. Chan et al., "Heritable somatic methylation and inactivation of $\mathrm{MSH} 2$ in families with Lynch syndrome due to deletion of the 3' exons of TACSTD1," Nature Genetics, vol. 41, no. 1, pp. 112-117, 2009.

[25] K. I. Kozaki, I. Imoto, S. Mogi, K. Omura, and J. Inazawa, "Exploration of tumor-suppressive microRNAs silenced by DNA hypermethylation in oral cancer," Cancer Research, vol. 68, no. 7, pp. 2094-2105, 2008.

[26] M. Furuta, K. I. Kozaki, S. Tanaka, S. Arii, I. Imoto, and J. Inazawa, "miR-124 and miR-203 are epigenetically silenced tumor-suppressive microRNAs in hepatocellular carcinoma," Carcinogenesis, vol. 31, no. 5, pp. 766-776, 2009.

[27] J. Pierson, B. Hostager, R. Fan, and R. Vibhakar, "Regulation of cyclin dependent kinase 6 by microRNA 124 in medulloblastoma," Journal of Neuro-Oncology, vol. 90, no. 1, pp. 1-7, 2008.

[28] Y. Saito, J. M. Friedman, Y. Chihara, G. Egger, J. C. Chuang, and G. Liang, "Epigenetic therapy upregulates the tumor suppressor microRNA-126 and its host gene EGFL7 in human cancer cells," Biochemical and Biophysical Research Communications, vol. 379, no. 3, pp. 726-731, 2009.

[29] H. Nakano, T. Miyazawa, K. Kinoshita, Y. Yamada, and T. Yoshida, "Functional screening identifies a microRNA, miR491 that induces apoptosis by targeting Bcl-XLin colorectal cancer cells," International Journal of Cancer, vol. 127, no. 5, pp. 1072-1080, 2010.

[30] Y. W. Huang, J. C. Liu, D. E. Deatherage et al., "Epigenetic repression of microRNA-129-2 leads to overexpression of SOX4 oncogene in endometrial cancer," Cancer Research, vol. 69, no. 23, pp. 9038-9046, 2009.

[31] T. Tsuruta, K. I. Kozaki, A. Uesugi et al., "miR-152 is a tumor suppressor microRNA that is silenced by DNA hypermethylation in endometrial cancer," Cancer Research, vol. 71, no. 20, pp. 6450-6462, 2011. 
[32] D. J. P. M. Stumpel, D. Schotte, E. A. M. Lange-Turenhout et al., "Hypermethylation of specific microRNA genes in MLL-rearranged infant acute lymphoblastic leukemia: major matters at a micro scale," Leukemia, vol. 25, no. 3, pp. 429439, 2011.

[33] Y. Chen, Y. Song, Z. Wang et al., "Altered expression of MiR$148 \mathrm{a}$ and MiR-152 in gastrointestinal cancers and its clinical significance," Journal of Gastrointestinal Surgery, vol. 14, no. 7, pp. 1170-1179, 2010.

[34] C. Braconi, N. Huang, and T. Patel, "Microrna-dependent regulation of DNA methyltransferase-1 and tumor suppressor gene expression by interleukin-6 in human malignant cholangiocytes," Hepatology, vol. 51, no. 3, pp. 881-890, 2010.

[35] H. Z. Chen, S. Y. Tsai, and G. Leone, "Emerging roles of E2Fs in cancer: an exit from cell cycle control," Nature Reviews Cancer, vol. 9, no. 11, pp. 785-797, 2009.

[36] L. Trusolino, A. Bertotti, and P. M. Comoglio, "MET signalling: principles and functions in development, organ regeneration and cancer," Nature Reviews Molecular Cell Biology, vol. 11, no. 12, pp. 834-848, 2010.

[37] D. Roulin, Y. Cerantola, A. Dormond-Meuwly, N. Demartines, and O. Dormond, "Targeting mTORC2 inhibits colon cancer cell proliferation in vitro and tumor formation in vivo," Molecular Cancer, vol. 9, article no. 57, 2010.

[38] D. A. Guertin, D. M. Stevens, M. Saitoh et al., "mTOR complex 2 is required for the development of prostate cancer induced by Pten loss in mice," Cancer Cell, vol. 15, no. 2, pp. 148-159, 2009. 

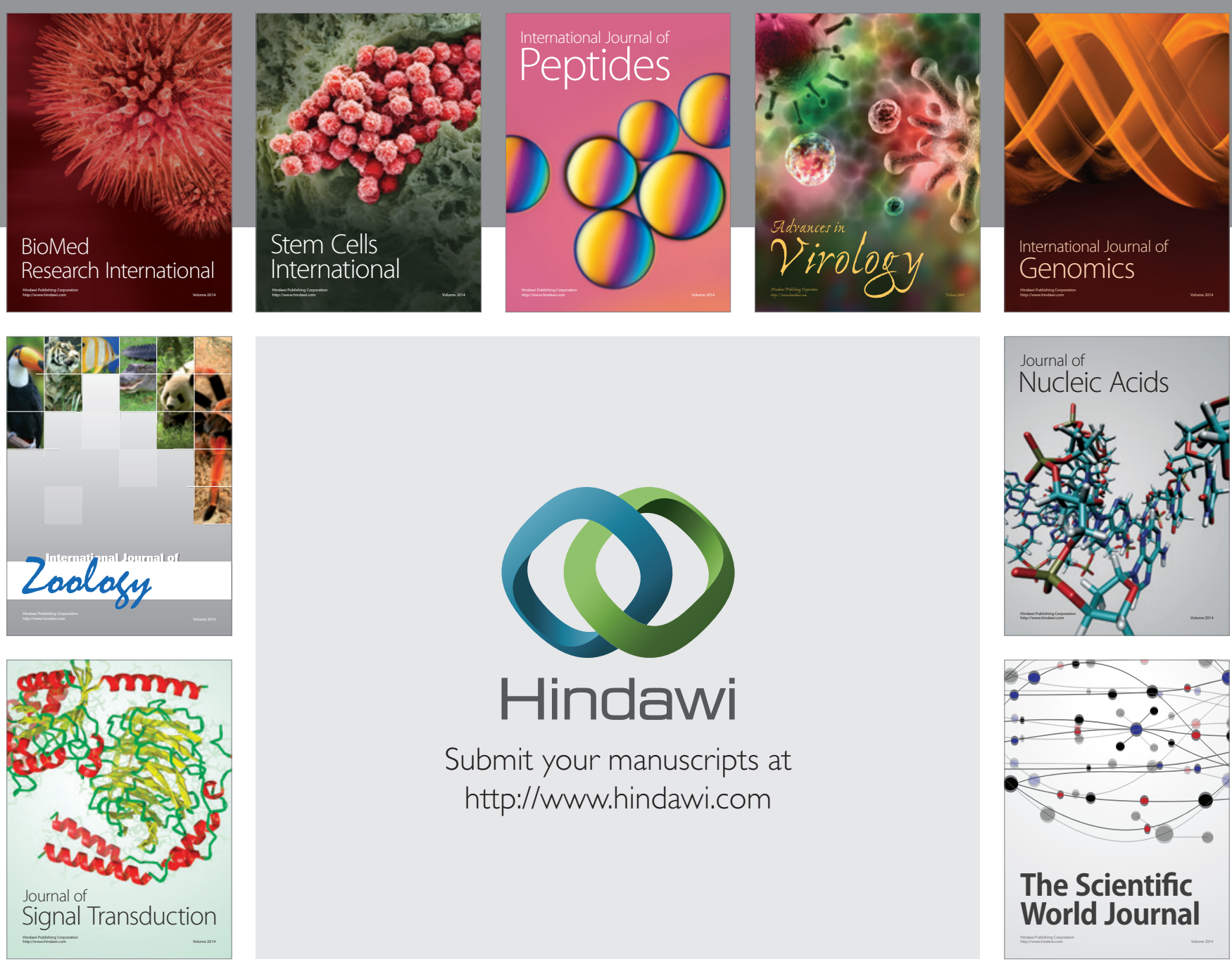

Submit your manuscripts at

http://www.hindawi.com
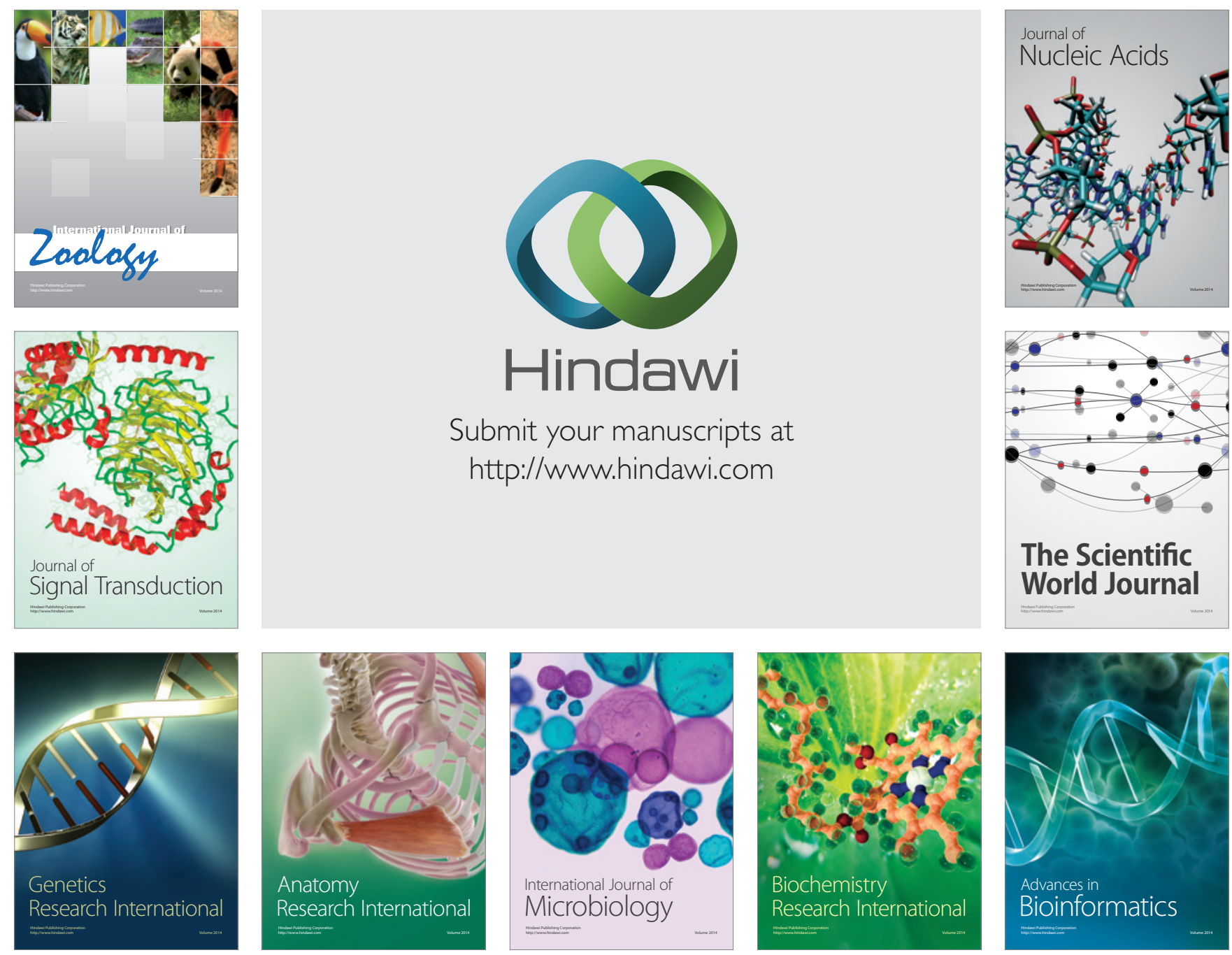

The Scientific World Journal
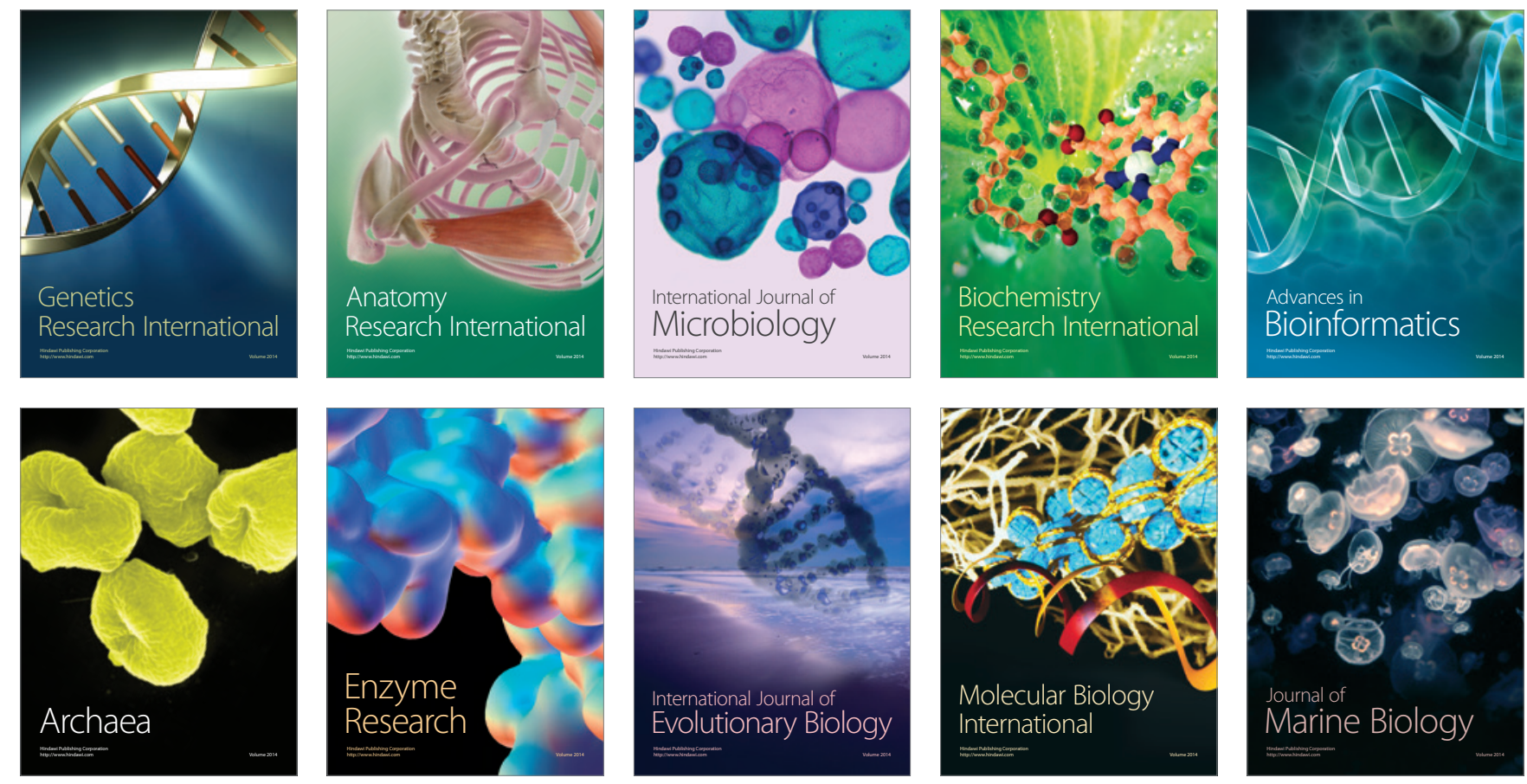